\section{Implementation of an Infection Control Bundle in a School to Reduce Transmission of Influenza-Like Illness during the Novel Influenza A 2009 H1N1 Pandemic}

To the Editor-We report the implementation of an infection control bundle to reduce transmission of novel influenza $\mathrm{A}$ $2009 \mathrm{H} 1 \mathrm{~N} 1$ virus in a Thai preschool. Prior interdisciplinary efforts to reduce the incidence of influenza-like illness (ILI) were associated with lower incidence of ILI at this same Thai preschool.' From May 1, 2009 through July 31, 2009, we prospectively studied the incidence of novel influenza A 2009 H1N1 and seasonal influenza among children at the Satit School of Rangsit University, a private school in central Thailand (Pratumthani). The intervention bundle was introduced in the kindergarten, and the primary school served as the control group. Components of the infection control bundle included (1) education about hand hygiene for preschool children, parents, and teachers, with an emphasis on beforeand-after activities (eg, use of toilets, diaper changes, and food preparation), by using cartoons and workshops to demonstrate the impact of hand hygiene on the transmission of respiratory tract and gastrointestinal tract infections; (2) education about cough etiquette for kindergarten children, parents, and teachers that emphasized use of this practice at both school and home, in accordance with the American Academy of Pediatrics Committee on Infectious Diseases; ${ }^{2}$ (3) compliance with an annual influenza vaccination day; and (4) enforcement of the school's rule to promote absenteeism of sick children. In addition, during the epidemic of novel influenza A $2009 \mathrm{H} 1 \mathrm{~N} 1$, kindergarten children were screened before daily school entry by measurement of temperature and inquiry about ILIs. Parents of children with fever or symptoms of ILI were contacted, and children were sent home until fever or ILI resolved.

Case detection of novel influenza A 2009 H1N1 was tracked by the school nurses, and all cases were confirmed by an infectious diseases physician (A.A.). In this study, the diagnosis of seasonal influenza was defined as a nasopharyngeal aspirate positive for seasonal influenza ( $\mathrm{H} 1$ or $\mathrm{H} 3$ ) or novel influenza A 2009 virus by reverse-transcriptase polymerase chain reaction. ${ }^{3,4}$ There was no attempt to identify other etiologies of respiratory tract infections. The intervention and control groups were compared for incidence of ILI, novel influenza A $2009 \mathrm{H} 1 \mathrm{Nl}$, and seasonal influenza. Novel influenza A $2009 \mathrm{H} 1 \mathrm{~N} 1$ incidence density was calculated and compared between groups with use of an incidence rate ratio.
There were 240 children in kindergarten (intervention group) and 289 children in the primary school (control group) during the study period; parents or guardians of all children provided consent, and all children were enrolled. The incidence of ILI was 7.1 cases per 1000 children-days among kindergarten children, compared with 14.9 cases per 1000 children-days among the primary school children (rate ratio, $2.4 ; 95 \%$ confidence interval, $1.14-5.77 ; P=.04$ ). Among the kindergarten children, there were no cases of novel influenza A H1N1 ( 0 cases per 1000 children-days) and 2 cases of seasonal influenza (H3N2; 0.14 cases per 1000 children-days). In contrast, in the primary school, there were 26 cases of novel influenza A H1N1 (2.64 cases per 1000 children-days; $P=.04$ ) and 13 cases of seasonal influenza (H3N2 and $\mathrm{H} 1 \mathrm{~N} 1 ; 1.3$ cases per 1000 children-days; $P=$ $.06)$.

Improvement of strategies to reduce the incidence of respiratory tract infection among school children has been especially important during the novel influenza A $2009 \mathrm{H} 1 \mathrm{~N} 1$ pandemic. Although school closure may limit transmission, such an intervention has broad societal implications beyond the school system. The Centers of Disease Control and Prevention suggests that "when contemplating school dismissals for influenza, officials should balance the goal of reducing exposure to $\mathrm{H} 1 \mathrm{~N} 1$ virus against the social disruption brought about by sending students home." Our study reinforces the simple maxims (ie, washing hands, covering the mouth and nose when coughing, receiving influenza vaccine, and staying home during illness) and suggests that effective implementation and reinforcement of these maxims can dramatically reduce the incidence of novel influenza $A 2009 \mathrm{H} 1 \mathrm{Nl}$ and seasonal influenza among preschool children. Because novel influenza A 2009 H1N1 virus cocirculates with seasonal influenza virus, our study findings support an emphasis the role of seasonal influenza vaccination among children during the novel influenza A $2009 \mathrm{H} 1 \mathrm{~N} 1$ pandemic.

\section{ACKNOWLEDGMENTS}

Financial support. National Center for Genetic Engineering and Biotechnology, National Science and Technology Development (BT-B-01-MG-135019 to A.A.).

Potentical conflicts of interest. All authors report no conflicts of interest relevant to this article.

Anucha Apisarnthanarak, MD; Piyaporn Apisarnthanarak, MD;

Boonsri Cheevakumjorn, $\mathrm{PhD}$; Linda M. Mundy, MD, PhD 
From the Division of Infectious Diseases, Thammasat University Hospital (A.A.), and Kindergarten Division, Satit School of Rangsit University (B.C.), Pratumthani, and Department of Radiology, Siriraj Hospital, Bangkok (P.A.), Thailand, and LM Mundy, LLC, Bryn Mawr, Pennsylvania (L.M.M.).

Address reprints request to Anucha Apisarnthanarak, MD, Division of Infectious Diseases, Thammasat University Hospital, Pratumthani, Thailand, 12120 (anapisarn@yahoo.com)

Infect Control Hosp Epidemiol 2010; 31:310-311

(C) 2010 by The Society for Healthcare Epidemiology of America. All rights reserved. 0899-823X/2010/3103-0015\$15.00. DOI: $10.1086 / 651063$

\section{REFERENCES}

1. Apisarnthanarak A, Apisarnthanarak P, Cheevakumjorn B, Mundy LM. Intervention with an infection control bundle to reduce transmission of influenza-like illnesses in a Thai preschool. Infect Control Hosp Epidemiol 2009; 30:817-822.

2. American Academy of Pediatrics Committee on Infectious Diseases. Infection prevention and control in pediatric ambulatory settings. Pediatrics 2007; 120:650-665.

3. Poddar SK. Influenza virus types and subtypes detection by single step single tube multiplex reverse transcription-polymerase chain reaction (RTPCR) and agarose gel electrophoresis. J Virol Methods 2002; 99:63-70.

4. CDC protocol of realtime RTPCR for influenza A (H1N1). Geneva: World Health Organization, April 2009. http://www.who.int/csr/resources/ publications/swineflu/CDCRealtimeRTPCR_SwineH1Assay-2009_2009 0430.pdf. Accessed July 1, 2009.

5. Centers for Disease Control and Prevention guidance for state and local public health officials and school administrators for school $(\mathrm{K}-12)$ responses to influenza during the 2009-2010 school year, August 2009. http: //www.pandemicflu.gov/plan/school/schoolguidance.html. Accessed August 10, 2009.

\section{Higher Incidence of Catheter-Related Bacteremia in Jugular Site with Tracheostomy than in Femoral Site}

To the Editor-Although the incidence of central venous catheter-related infection has been the objective of many studies, ${ }^{1-9}$ we have not found data about the incidence in which the jugular site with tracheostomy is compared with the femoral site. Some studies found a higher incidence of central venous catheter-related infection ${ }^{1,2}$ in jugular and subclavian sites with the presence of tracheostomy than without tracheostomy. A higher incidence of central venous catheter-related infection in the femoral site than in the jugular site was found in several studies; ${ }^{3-6}$ however, other studies found a higher incidence in the jugular than the femoral site. ${ }^{7.9}$ However, none of these studies reported the rate of tracheostomy. The recently published guidelines "Strategies to Prevent Central Line-Associated Bloodstream Infections in Acute Care Hospitals" by the Society for Healthcare Epidemiology of America/Infectious Diseases Society of America ${ }^{10}$ recommend avoiding femoral access; however, there are no recommen- dations about catheter site with regard to the presence of tracheostomy. Thus, we designed a study to determine whether the jugular site with tracheostomy may have a higher risk of catheter-related bacteremia, compared with the femoral site. This prospective observational study was performed May 1 , 2000, to April 30, 2004, at the Intensive Care Unit of the University Hospital of the Canary Islands (Santa Cruz de Tenerife, Spain). The study was approved by the institutional review board.

Catheter-related bacteremia was defined according to the following criteria: positive culture result of a blood sample obtained from a peripheral vein; signs of systemic infection (eg, fever, chills, or hypotension), with no apparent source of bacteremia except a central venous catheter; and cathetertip colonization (growth of a microorganism of more than 15 CFU from the central venous catheter tip) with the same organism as in blood culture (ie, the same species with identical antimicrobial susceptibility).

Cases of central venous catheter-related bacteremia in patients with tracheostomy were recorded if all of the following criteria were met: (1) presence of the same microorganism in a tracheal aspirate specimen (concentration, $<10^{6} \mathrm{CFU} /$ $\mathrm{mL}$ ) as in a catheter-tip specimen and in a blood specimen obtained from a peripheral vein, (2) absence of purulent tracheal aspirate, and (3) absence of a new or progressive pulmonary infiltrate on chest radiographs. Thus, bacteremia in the presence of the same microorganism in tracheal aspirate and blood cultures and in the presence of tracheobronchitis or pneumonia was considered to be secondary to the respiratory origin and not to the catheter.

Statistical analyses were performed with SPSS software, version 12.0.1 (SPSS); LogXact, version 4.1 (Cytel); and StatXact, version 5.0.3 (Cytel). Continuous variables are reported as means and standard deviations, and categorical variables are reported as frequencies and percentages. Comparisons between groups for continuous variables were performed using the Wilcoxon-Mann-Whitney test. Comparisons between groups for categorical variables were performed using the Kruskall-Wallis test for singly ordered rows $\times$ columns $(\mathrm{R} \times \mathrm{C})$ tables. The comparison of the incidence of catheterrelated bacteremia per 1000 catheter-days between groups was performed using the risk ratio of catheter-related bacteremia and its $95 \%$ confidence interval. $P$ values less than .05 were considered to be statistically significant.

We diagnosed 16 cases of catheter-related bacteremia involving 208 femoral catheters during 1679 days of catheterization; the incidence of catheter-related bacteremia was 9.52 episodes per 1000 days of risk. We diagnosed 10 cases of catheter-related bacteremia involving 52 central internal jugular catheters during 462 days of catheterization; the incidence of catheter-related bacteremia was 21.64 episodes per 1000 days of risk.

There were no significant differences in the baseline characteristics of both patient groups (Table). Poisson regression 